\title{
Exploiting Quantum Parallelism To Simulate Quantum Random Many-Body Systems
}

\author{
B. Paredes, ${ }^{1}$ F. Verstraete ${ }^{2}$ and J. I. Cirac ${ }^{1}$ \\ ${ }^{1}$ Max-Planck Institut für Quantenoptik, Hans-KopfermannStr. 1, Garching, D-85748 Germany \\ ${ }^{2}$ Institute for Quantum Information, Caltech, Pasadena 91125, CA
}

(Dated: October 16, 2018)

\begin{abstract}
We present an algorithm that exploits quantum parallelism to simulate randomness in a quantum system. In our scheme, all possible realizations of the random parameters are encoded quantum mechanically in a superposition state of an auxiliary system. We show how our algorithm allows for the efficient simulation of dynamics of quantum random spin chains with known numerical methods. We also propose an experimental realization based on atoms in optical lattices in which disorder could be simulated in parallel and in a controlled way through the interaction with another atomic species.

PACS numbers: 03.75.Fi, 03.67.-a, 42.50.-p, 73.43.-f
\end{abstract}

One of the most remarkable features of quantum mechanics is that it allows for the creation of superposition states, making the ambitious dream of performing many tasks at the same time a real possibility. This extraordinary concession lies at the heart of quantum computation and is the basis of all quantum algorithms developed so far 11. In this letter we present a new algorithm that exploits this quantum parallelism to simulate in parallel many different evolutions of a quantum system. Our motivation is the simulation of physical systems whose understanding requires to study their behavior under many different Hamiltonians. An important example of such systems are quantum random systems (QRS) 2]. For them certain parameters of the Hamiltonian (e.g., interaction couplings, potential strengths) are random (classical) variables. Therefore the exact simulation of their dynamics requires to perform many evolutions, one for each realization of the set of random variables. QRS have captured a lot of attention in the last decades [2, 3, 4]. The presence of randomness can dramatically change the behavior of quantum many-body systems, leading to fascinating phenomena 2]. Moreover, the answer to puzzles as the unusual transport properties of high temperature superconductor materials is inextricably tied to the understanding of phase transitions and transport in the presence of disorder [5]. On the theoretical side, the understanding of QRS is hindered by the fact that the number of required simulations for an exact calculation scales exponentially with the number of random parameters $[\underline{6}]$. On the experimental side, one of the big challenges is the creation of randomness in a controlled way. Here, atomic systems in optical lattices [7, 8], highly versatile and controllable, are one of the most promising candidates.

In this letter we present an algorithm that allows to simulate dynamics (and ground state properties) of a QRS within one single time evolution in which the system is put into interaction with an auxiliary system. The key idea is that all possible realizations of the set of random classical parameters are encoded quantum mechanically in a superposition state of the auxiliary system. Choosing the interaction with the ancilla in the appropriate way, all possible quantum evolutions of the QRS are simulated in parallel. As a particular case, adiabatic evolution with the ancilla can simulate at once all possible ground states of the QRS. As one of the main results of this work, our algorithm establishes an exact mapping between a QRS and a certain interacting (non-random) system. This equivalence opens a new path both to the numerical and experimental simulation of QRS. On the numerical side, it allows for the efficient simulation of QRS within the framework of numerical methods that simulate the corresponding interacting systems efficiently. For example, for the case of a quantum random spin chain we will show that the problem is mapped onto the simulation of the time evolution of a one-dimensional (1D) lattice system. Recently, numerical methods inspired in density matrix renormalization group (DMRG) $\underline{9}]$ and matrix-productstate (MPS) 10] techniques have been developed to efficiently simulate the time evolution of 1D lattice systems [11, 12, 13]. Here we will show how to implement the efficient simulation of quantum random spin chains within the methods introduced in [12]. On the experimental side our scheme opens the possibility of simulating randomness in parallel through the interaction with an auxiliary quantum system. Moreover, conversely, it allows to interpret current interacting experimental schemes as potential simulators of certain random equivalent problems. We propose an experimental scheme in which a variety of disordered phases as quantum glasses [14, 15] or Anderson insulator phases 16 could be simulated in current experiments with optical lattices [17, 18].

The algorithm. Let us consider a quantum system with Hilbert space $\mathcal{H}$ that evolves accordingly to a Hamiltonian $H\left(r_{1}, \ldots, r_{n}\right)$ where $r_{1}, \ldots, r_{n}$ are random variables that take values within a finite discrete set, $r_{\ell} \in$ $\Gamma_{\ell}=\left\{\lambda_{1}^{\ell}, \ldots \lambda_{m_{\ell}}^{\ell}\right\}$, with a probability distribution given by $p\left(r_{1}, \ldots, r_{n}\right)$. In order to simulate exactly the dynamics of such a system one would need to perform $\prod_{\ell=1}^{n} m_{\ell}$ simulations, one per each possible realization of the set of random variables $\mathbf{r}=\left(r_{1}, \ldots r_{n}\right)$. For 
each realization the system evolves to a different state $\left|\psi_{\mathbf{r}}(t)\right\rangle=e^{-i H(\mathbf{r}) t / \hbar}\left|\psi_{0}\right\rangle$, where $\left|\psi_{0}\right\rangle$ is the initial state. Given this set of evolved states and a physical observable $\hat{O}$, one is typically interested in the average of the expectation values of that observable in the different evolved states, that is, in quantities of the form:

$$
\langle\langle\hat{O}(t)\rangle\rangle:=\sum_{\mathbf{r}} p(\mathbf{r})\left\langle\psi_{\mathbf{r}}(t)|\hat{O}| \psi_{\mathbf{r}}(t)\right\rangle .
$$

On the following we describe an algorithm that allows to simulate in parallel all possible time evolutions of the random system described above. We consider an auxiliary system with Hilbert space $\mathcal{H}_{a}$ and a Hamiltonian acting on $\mathcal{H} \otimes \mathcal{H}_{a}$ of the form $\widetilde{H}=H\left(\hat{R}_{1}, \ldots, \hat{R_{n}}\right)$, where $\hat{R}_{1}, \ldots, \hat{R_{n}}$ are operators that act in $\mathcal{H}_{a}$, commute with each other and have spectra $\Gamma_{1}, \ldots, \Gamma_{n}$. Note that we have replaced the set of random variables $\mathbf{r}$ by a set of quantum operators $\hat{\mathbf{R}}$ with the same spectra. The algorithm works as follows. 1) Initialization. Let us prepare the auxiliary system in an initial superposition state of the form:

$$
\left|\psi_{a}\right\rangle=\sum_{\mathbf{r}} \sqrt{p(\mathbf{r})}|\mathbf{r}\rangle
$$

where the states $|\mathbf{r}\rangle$ are simultaneous eigenstates of the set of operators $\hat{\mathbf{R}}$, with $\hat{R}_{\ell}|\mathbf{r}\rangle=r_{\ell}|\mathbf{r}\rangle$. Each state $|\mathbf{r}\rangle$ is therefore in one to one correspondence with one realization of the the set of random variables $\mathbf{r}$, its weight in the superposition state (2) being equal to the probability with which the corresponding realization occurs for the random system. 2) Evolution. We evolve the initial state of the composite system $\left|\psi_{0}\right\rangle \otimes\left|\psi_{a}\right\rangle$ under the Hamiltonian $\widetilde{H}$. The evolved state is

$$
|\Psi(t)\rangle=\sum_{\mathbf{r}} \sqrt{p(\mathbf{r})}\left|\psi_{\mathbf{r}}(t)\right\rangle \otimes|\mathbf{r}\rangle .
$$

This superposition state contains the complete set of evolved states we are interested in. 3) Read-out. In order to obtain the quantities (1) we just need to measure the observable $\hat{O} \otimes 1$,

$$
\langle\Psi(t)|\hat{O} \otimes 1| \Psi(t)\rangle=\langle\langle\hat{O}(t)\rangle\rangle .
$$

The algorithm above allows us, in particular, to obtain the averaged properties of a random system over the collection of all possible ground states. Let us assume that the interaction between the system and the ancilla is introduced adiabatically, so that the Hamiltonian is now $\widetilde{H}(t)=H(\beta(t) \hat{\mathbf{R}})$, where $\beta(t)$ is a slowly varying function of time with $\beta(0)=0, \beta(T)=1, T$ being the time duration of the evolution. If the system is prepared in the ground state of the Hamiltonian $H(\mathbf{0})$, the algorithm above will simulate in parallel all possible adiabatic paths, so that the composite superposition state (3) will contain all possible ground states of the random system [19].
Additionally, the scheme above can be easily extended for the computation of other moments of the distribution of physical observables (higher than (10), which are sometimes important in the understanding of QRS [4]. For example, quantities like $\left\langle\left\langle\hat{O}^{2}\right\rangle-\langle\hat{O}\rangle^{2}\right\rangle$, can be computed by using an additional copy of the system [20].

Numerical implementation. The algorithm described above reduces the simulation of a quantum random system to the simulation of an equivalent non-random interacting problem. This exact mapping allows us to integrate the simulation of randomness in quantum systems within the framework of numerical methods that are able to efficiently simulate the corresponding interacting problem. As an illustrative example we consider the case of a $1 \mathrm{D}$ spin $s=1 / 2$ system with random local magnetic field. The Hamiltonian of the system is:

$$
H\left(b_{1}, \ldots, b_{N}\right)=H_{0}+B \sum_{\ell=1}^{N} b_{\ell} S_{\ell}^{z},
$$

where $H_{0}$ is a short range interaction Hamiltonian, $\mathbf{b}=$ $\left(b_{1}, \ldots, b_{N}\right)$ is a set of classical random variables that take values $\{1 / 2,-1 / 2\}$ with probability distribution $p(\mathbf{b})$. Following the algorithm above the $2^{N}$ simulations required for the exact simulation of the dynamics (or the ground-state properties) of this random problem can be simulated in parallel as follows. We consider an auxiliary $1 \mathrm{D}$ spin $\sigma=1 / 2$ system. We prepare this ancilla in the initial state $\left|\psi_{a}\right\rangle=\sum_{\mathbf{b}} \alpha_{\mathbf{b}}|\mathbf{b}\rangle$, where the states $|\mathbf{b}\rangle$ have all $z$ components of the $N$ spins well defined, $\widehat{\sigma}_{\ell}^{z}|\mathbf{b}\rangle=b_{\ell}|\mathbf{b}\rangle$, and $\alpha_{\mathbf{b}}=\sqrt{p(\mathbf{b})}$. The entangled properties of the state of the ancilla reflect the classical correlations among the random variables. For example, for a uniform distribution of the random field, $p(\mathbf{b})=1 / 2^{N}$, the state of the ancilla is just a product state, $\left|\psi_{a}\right\rangle \propto(|\uparrow\rangle+|\downarrow\rangle)^{\otimes N}$. We evolve the system and the ancilla under the interaction Hamiltonian

$$
\widetilde{H}=H\left(\widehat{\sigma}_{1}^{z}, \ldots, \widehat{\sigma}_{n}^{z}\right)=H_{0}+\beta \sum_{\ell} \widehat{\sigma}_{\ell}^{z} \widehat{S}_{\ell}^{z} .
$$

Here, $\beta=B$ if we want to simulate dynamics under Hamiltonian (5), and $\beta$ is a slowly varying function of time with $\beta(0)=0$ and $\beta(T)=B$ for the simulation of the ground state properties. We have then reduced the simulation of the random problem to that of the time evolution of two coupled spin $1 / 2$ chains with Hamiltonian (6). This problem is equivalent to a $1 \mathrm{D}$ lattice problem of $N$ sites with physical dimension $d=2 \times 2$, which can be easily incorporated to the framework of the numerical methods introduced in 11, 12]. Implementation of the scheme above is as follows.

1) Let the state $|\Psi(0)\rangle$ be the MPS with dimension $D$ that better approximates the initial state $\left|\psi_{0}\right\rangle \otimes\left|\psi_{a}\right\rangle$, $|\Psi(0)\rangle=\sum_{s_{1}, \ldots, s_{N}=1}^{d} \operatorname{Tr}\left(A_{1}^{s_{1}} \ldots A_{N}^{s_{N}}\right)\left|s_{1} \ldots s_{N}\right\rangle$. Here, the $A$ 's are matrices whose dimension is bounded by $D$ and $d=4$. 2) We evolve $|\Psi(0)\rangle$ under Hamiltonian (6). As in [12] we take a small time step $\Delta t$ and compute 
$|\Psi(\Delta t)\rangle$ exactly, a state for which the dimension of the matrices $A$ will be typically larger. Following 12 we then "truncate" the matrices in an optimal way and use the "truncated" state to compute the next time step. 3) At any time $t$ we can efficiently determine the quanti-
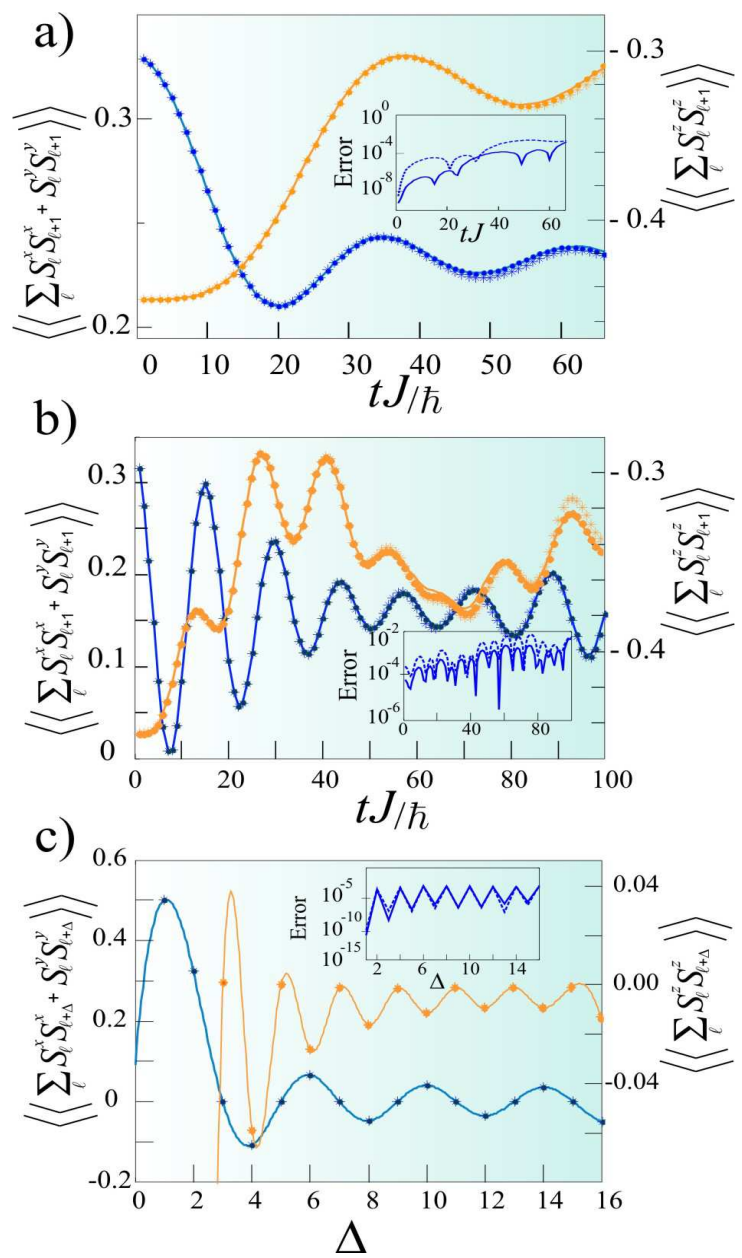

FIG. 1: Comparison of our numerical simulation of a random field XY spin chain with an exact calculation. We plot the exact results (full line) together with the numerical results using MPS with $D=20$ (circles) and $D=30$ (stars). The relative errors are plotted in the insets. Figures (a) and (b) show the time evolution of the averaged correlations $\left\langle\left\langle\sum_{\ell} S_{\ell}^{x} S_{\ell+1}^{x}+S_{\ell}^{y} S_{\ell+1}^{y}\right\rangle\right\rangle$ (blue) and $\left\langle\left\langle\sum_{\ell} S_{\ell}^{z} S_{\ell+1}^{z}\right\rangle\right\rangle$ (orange). The evolution Hamiltonian is (5) with (a) $B_{0}=0$, $J / B=-2$ and $p(\mathbf{b})=1 / 2^{N}$, and (b) $B_{0}=0, J / B=-4$ and $p(\mathbf{b})=\left|\alpha_{\mathbf{b}}\right|^{\mathbf{2}}$, where $\alpha_{\mathbf{b}}$ are given by $\left|\psi_{a}\right\rangle=\sum_{\mathbf{b}} \alpha_{\mathbf{b}}|\mathbf{b}\rangle$, and $\left|\psi_{a}\right\rangle$ is the ground state of $H_{0}$ with $B_{0} / J=1.4$. For both figures the chain is prepared initially in the ground state of $H_{0}$ with $B_{0}=0$. The time step for the numerical simulations is $\Delta t=0.01 J / \hbar$. Fig. (c) shows the correlation functions $\left\langle\left\langle\sum_{\ell} S_{\ell}^{x} S_{\ell+\Delta}^{x}+S_{\ell}^{y} S_{\ell+\Delta}^{y}\right\rangle\right\rangle$ (blue) and $\left\langle\left\langle\sum_{\ell} S_{\ell}^{z} S_{\ell+\Delta}^{z}\right\rangle\right\rangle$ (orange) averaged over all possible ground states of Hamiltonian (5) for $B_{0}=0, J / B=-1$ and $p(\mathbf{b})=1 / 2^{N}$ as a function of the distance $\Delta$ between spins. The numerical simulation performs an adiabatic evolution with Hamiltonian (6) with $\beta(t)=B t / T$ and $T=100 J / \hbar$. ties $\left\langle\left\langle O_{1} \ldots O_{N}\right\rangle\right\rangle$ as $\left\langle O_{1} \otimes 1 \ldots O_{N} \otimes 1\right\rangle$, which can be efficiently computed for MPS [12].

In order to test the efficiency of the simulation scheme above we have compared it with an exact calculation for the case in which $H_{0}$ is an XY model Hamiltonian, $H_{0}=-J \sum_{\ell=1}^{N} S_{\ell}^{x} S_{\ell+1}^{x}+S_{\ell}^{y} S_{\ell+1}^{y}+B_{0} \sum_{\ell=1}^{N} S_{\ell}^{z}$. The exact averages (11) are calculated in the following way. For each realization of the magnetic field the evolved state (or ground state) of the Hamiltonian (5) is computed exactly using fermionization techniques 21, 22]. The quantities (11) are then determined by averaging over the expectation values of the $2^{N}$ states obtained. The comparison is shown in Fig. 1 for $N=16$ and different correlations functions averaged over the collection of evolved states (Fig. 1(a) and 1(b)) and ground states (Fig. 1(c)), both for a uniform probability distribution (Fig. 1(a)) and a correlated one (Fig. 1(b)). Using MPS with $D=20$ we obtain a very good accuracy (see insets in Fig. 1). As in [11, 12] we have two sources of error. One is the Trotter expansion. Here the error can be decreased by considering smaller time steps. The other source of error is the truncation of the MPS to a smaller dimension. For the simulations we have performed this error did not grow much with the size of the system. Using the numerical scheme above we have performed simulations of the dynamics of random field XY (Fig. 2) and Heisenberg (Fig. 3) chains with $N=40$.

The scheme above can be easily extended for the nu-

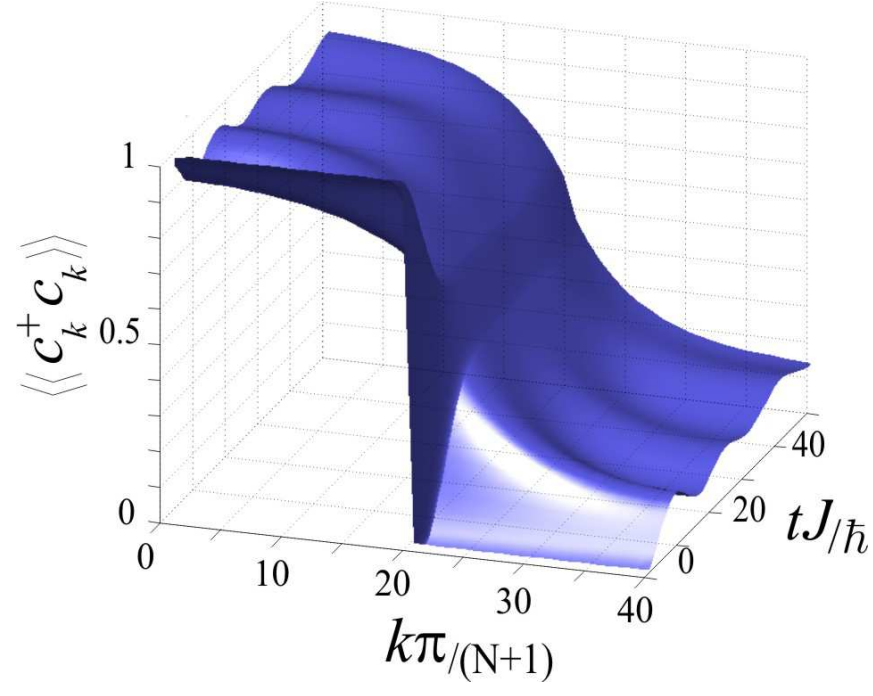

FIG. 2: Numerical simulation of the time evolution of a random field XY spin chain with $N=40$. We show the correlation function $\left\langle\left\langle c_{k}^{\dagger} c_{k}\right\rangle\right\rangle$ as a function of time and momentum $k$. Here $c_{k} \propto \sum_{\ell} \sin (k \ell) \widetilde{c}_{\ell}, k=\frac{\pi}{N+1}, \ldots, \frac{\pi N}{N+1}$ and $\widetilde{c}_{\ell}=\prod_{\ell<\ell^{\prime}} S_{\ell^{\prime}}^{z}\left(S_{\ell}^{x}+i S_{\ell}^{y}\right)$ are the fermionic operators given by the Jordan-Wigner transformation [21]. The evolution Hamiltonian is (5) with $H_{0}$ being the XY Hamiltonian with $B_{0}=0, B / J=-4$ and $p(\mathbf{b})=1 / 2^{N}$. The initial state $\left|\psi_{0}\right\rangle$ is the ground state of $H_{0}$. As the system evolves in time the initially sharp Fermi sea disappears. 


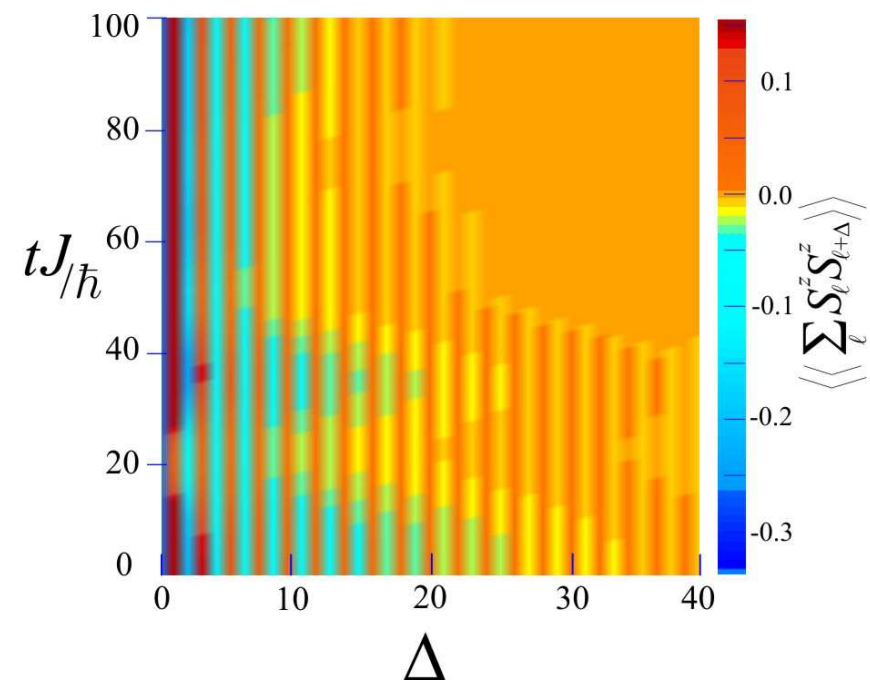

FIG. 3: Numerical simulation of the time evolution of a random field Heisenberg spin chain with $N=40$. We show the correlation function $\left\langle\left\langle\sum_{\ell} S_{\ell}^{z} S_{\ell+\Delta}^{z}\right\rangle\right\rangle$ as a function of time and the separation $\Delta$ between the spins. The evolution Hamiltonian is (5) with $H_{0}$ being the antiferromagnetic Heisenberg Hamiltonian, $B / J=-4$ and $p(\mathbf{b})=1 / 2^{N}$. The initial state $\left|\psi_{0}\right\rangle$ is the ground state of $H_{0}$. As the system evolves in time the antiferromagnetic correlations are smeared out.

merical simulation of spin chains with random couplings. For example, for the case of a Heisenberg chain with Hamiltonian $H\left(J_{1}, \ldots, J_{N}\right)=\sum_{\ell} J_{\ell} \mathbf{S}_{\ell} \cdot \mathbf{S}_{\ell+1}$, the problem is mapped to the simulation of the time evolution of two spin chains under the three-body interaction Hamiltonian $\widetilde{H}=H\left(\widehat{\sigma}_{1}^{z}, \ldots, \widehat{\sigma}_{n}^{z}\right)=\sum_{\ell} \widehat{\sigma}_{\ell}^{z} \mathbf{S}_{\ell} \cdot \mathbf{S}_{\ell+1}$.

Experimental proposal. Using the ideas of the algorithm above we present an experimental scheme for atoms in optical lattices that could be use as a simulation protocol for a variety of disordered phases. We consider a system of atoms $b$ (bosons or fermions) in an optical lattice (3D, 2D, or 1D) in a certain state $\left|\psi_{0}\right\rangle$. We consider another system of atoms $a$ (e.g, another spin state or atomic species) which experiences an independent lattice potential 23]. We consider a situation in which the lattice potentials of atoms $a$ and $b$ are initially shifted in such a way that there is no interaction between the two systems. We proceed as follows: 1) We prepare atoms $a$ is a certain state $\left|\psi_{a}\right\rangle$. This state can always be written in a Fock basis as $\left|\psi_{a}\right\rangle=\sum_{n_{1} \ldots n_{M}} \alpha_{n_{1}, \ldots n_{M}}\left|n_{1} \ldots n_{M}\right\rangle$, where $n_{1}, \ldots n_{M}$ are the occupation numbers of the $M$ lattice sites and the $\alpha$ 's are certain complex coefficients. We then suddenly ramp up the lattice for atoms $a$ (so that tunneling processes are instantaneously suppresed), and shift it so that the interaction with atoms $b$ is instantaneously switched on. 2) We let the composite system evolve. The Hamiltonian that governs the evolution is

$$
\widetilde{H}=H_{b}+U_{a b} \sum_{\ell=1}^{M} \hat{n}_{\ell}^{a} \hat{n}_{\ell}^{b}
$$

where $H_{b}$ is the Hubbard Hamiltonian for atoms $b, \hat{n}_{\ell}^{a}, \hat{n}_{\ell}^{b}$ are the local density operators for atoms $a$ and $b$, and $U_{a b}$ is the interaction coupling between atoms $a$ and $b$. 3) We finally measure the system $b$. According to the algorithm developed above, this interacting experimental scheme is simulating in parallel all possible dynamics of atoms $b$ under the random Hamiltonian:

$$
H\left(V_{1}, \ldots, V_{M}\right)=H_{b}+U_{a b} \sum_{\ell=1}^{M} V_{\ell} \hat{n}_{\ell}^{b},
$$

where $V_{1}, \ldots, V_{M}$ are random potential strengths that take values within $\left\{0,1, \ldots, N_{a}\right\}$ with a probability distribution given by $p\left(V_{1}, \ldots, V_{M}\right)=\left|\alpha_{V_{1}, \ldots V_{N}}\right|^{2}, N_{a}$ being the number of $a$ atoms. Choosing the initial state of atoms $a$ in the appropriate way we can tune the probability distribution of the random potential for atoms $b$. Changing the entanglement properties of the state in which atoms $a$ are prepared we will change the correlation properties among the random local potentials. As well, the intensity of the disorder potential can be tuned by varying the interaction strength $U_{a b}$ (e.g., shifting the lattices). This allows to simulate a large variety of disordered phases. As opposite to the classical simulation of randomness (e.g.,with speckle lasers 24]) our quantum mechanical approach allows to simulate all possible evolutions of the random system in one single run of the experiment. Concerning measurements, note that in an experiment we will typically have many copies of the system (an array of $2 \mathrm{D}$ or 1D systems) so that the outcomes will be already averaged over all copies. As an example of current interest let us consider the case in which atoms $a$ and $b$ are initially prepared in two independent Tonks states [22] with filling factor $\nu_{a}$ and $\nu_{b}$. For this case

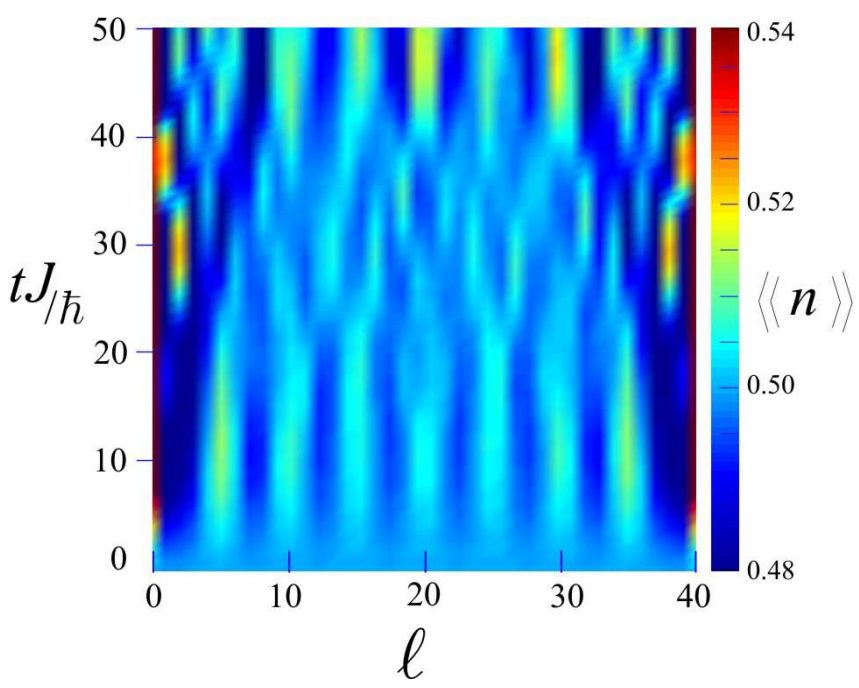

FIG. 4: Numerical simulation of the time evolution of the averaged density of a Tonks gas in an optical lattice with $M=40$ sites and $\nu=1 / 2$ in the random potential generated by another Tonks gas with $\nu=1 / 5$. 
the experimental scheme above would simulate the dynamics of a Tonks gas in the presence of the random potential generated by another Tonks gas. Using the numerical scheme above we have simulated this situation for $M=40$ sites and $\nu_{b}=1 / 2, \nu_{a}=1 / 5$. Interestingly, the averaged density of atoms $b$ shows localization of atoms $b$ in the regions in which atoms $a$ are most probably absent (see Fig. 4).

In conclusion, we have presented an algorithm that al- lows to simulate (classical) randomness in quantum many body systems via a single quantum mechanical problem in which quantum interactions allow all possible quantum paths of the random system to occur simultaneously. Our scheme opens new possibilities in the numerical and experimental simulation of QRS.

We thank M. A. Martín-Delgado for helpful discussions. Work supported in part by DFG, EU projects and Bayerische Staatsregierung.
[1] See for example C. H. Bennett, Nature 362, 694 (1993).

[2] See D. S. Fisher, G. M. Grinstein, A. Khurana, Physics Today, December 1988, 56, and references there in.

[3] D. Belitz, T. R. Kirkpatrick, Rev. Mod. Phys. 66, 261 (1994).

[4] D. S. Fisher, Phys. Rev. B 51, 6411 (1995).

[5] D. A. Huse, M. P. A. Fisher, D. S. Fisher, Nature 358, 553 (1992).

[6] Asymptotically exact results can be nevertheless obtained with real renormalization-group treatments. See [4] and G. Refael, D. S. Fisher, Phys. Rev. B 70, 064409 (2004).

[7] I. Bloch, Physics World 1725 (2004).

[8] J. I. Cirac, P. Zoller, Physics Today, March 2004, 38.

[9] S. R. White, Phys. Rev. Lett. 69, 2863 (1992).

[10] S. Ostlund, S. Rommer, Phys. Rev. Lett. 75, 3537 (1995).

[11] G. Vidal, Phys. Rev. Lett. 91, 147902 (2003).

[12] F. Vertraete, J. J. Garca-Ripoll, J. I. Cirac, Phys. Rev. Lett. 93, 207204 (2004).

[13] S. R. White, A. E. Feiguin, Phys. Rev. Lett. 93, 076401 (2004), A. Daley et al., J. Stat. Mech,: Theor. Exp. P04005 (2004).

[14] M. P. A. Fisher et al. Phys. Rev. B 40, 546 (1989).

[15] A. Sanpera, A. Kantian, L. Sánchez-Palencia, J. Za- krzewski, M. Lewenstein, Phys. Rev. Lett. 93, 040401 (2004).

[16] K. Byczuk, W. Hofstetter, D. Vollhardt, Phys. Rev. Lett. 94, 056404 (2005).

[17] M. Greiner et al., Nature 415, 39 (2002).

[18] M. Köhl, H. Moritz, T. Stöferle, K. Günter, T. Esslinger, Phys. Rev. Lett. 94, 080403 (2005).

[19] As long as there are no level crossings.

[20] Let us consider two independent copies of the system both interacting with the ancilla via the Hamiltonian $\widetilde{H}^{(1)}+\widetilde{H}^{(2)}$. The 3-party system evolves then to the state $|\Psi(t)\rangle=\sum_{\mathbf{r}} \sqrt{p(\mathbf{r})}\left|\psi_{\mathbf{r}}(t)\right\rangle^{(1)} \otimes\left|\psi_{\mathbf{r}}(t)\right\rangle^{(2)} \otimes|\mathbf{r}\rangle$. We then measure $\left\langle\Psi(t)\left|\hat{O}^{(1)} \otimes \hat{O}^{(2)} \otimes 1\right| \Psi(t)\right\rangle=\left\langle\langle\hat{O}\rangle^{2}\right\rangle$.

[21] S. Sachdev, Quantum Phase Transitions (Cambridge University Press, Cambridge, 1999).

[22] B. Paredes, A. Widera, V. Murg, O. Mandel, S. Fölling, I. Cirac, G. V. Shlyapnikov, T. W. Hänsch and I. Bloch, Nature 429, 277 (2004).

[23] O. Mandel, M. Greiner, A. Widera, T. Rom, T.W. Hänsch, and I. Bloch, Phys. Rev. Lett. 91, 010407 (2003).

[24] P. Horak, J. Y. Courtois, and G. Grynberg, Phys. Rev. A 58, 3953 (1998). 